\title{
Innovations in implantation techniques of ventricular assist devices
}

Jasmin S. Hanke, MD, Günes Dogan, MD, Malakh Shrestha, MD, PhD, Axel Haverich, MD, PhD, and Jan D. Schmitto, MD, PhD

Innovation is defined as the production, adoption, or integration of a value-added novelty. ${ }^{1}$ The most common definitions of innovations describe 4 subtypes in which innovations can be categorized: (1) basic research, (2) breakthrough innovation, (3) sustaining innovation, and (4) disruptive innovation. ${ }^{1,2}$

Technological and surgical innovations are greatly interdependent, and some medical specialties, such as mechanical circulatory support (MCS) systems, are especially technology-driven. Habitually innovative developments, such as the miniaturization of devices, lead to the establishment of new implantation strategies. Contrarily, the constant longing of physicians for solutions for surgical or clinical challenges and patients' preferences drive engineers to adapt and reinvent technological devices.

A breakthrough innovation in the development of MCS was the shift from pulsatile-flow to continuous-flow pumps, which facilitated the miniaturization of ventricular assist devices (VADs). ${ }^{3}$ Whereas the early versions of these devices required extended sternotomy owing to their bulky size, the new generation of devices has reduced the invasiveness of the implantation procedure and has facilitated less invasive implantation strategies as a sustaining innovation. ${ }^{3-6}$ The combination of advanced technological devices, improved implantation techniques, and reduced adverse events rates contributed greatly to the wide acceptance of the new generation of VADs and their permanent inclusion in the treatment guidelines for terminal heart failure. ${ }^{7}$

In this article, we discuss these innovative approaches, as well as alternative surgical approaches, for VAD implantation and explore possible future developments.

\section{Less Invasive VAD Implantation Through Thoracotomy}

In the past, the most frequently used technique for VAD implantation was a median sternotomy, a well-established

\footnotetext{
From the Department of Cardiac, Thoracic, Transplantation, and Vascular Surgery, Hannover Medical School, Hannover, Germany.

Received for publication July 30, 2021; accepted for publication Sept 27, 2021.

Address for reprints: Jasmin S. Hanke, MD, Department of Cardiac, Thoracic, Transplantation and Vascular Surgery, Hannover Medical School, Carl-Neuberg Str 1, 30625 Hannover, Germany (E-mail: Hanke.Jasmin@mh-hannover.de). JTCVS Open 2021;8:28-32

2666-2736

Copyright (C 2021 The Author(s). Published by Elsevier Inc. on behalf of The American Association for Thoracic Surgery. This is an open access article under the CC BY-NC-ND license (http://creativecommons.org/licenses/by-nc-nd/4.0/). https://doi.org/10.1016/j.xjon.2021.09.047
}
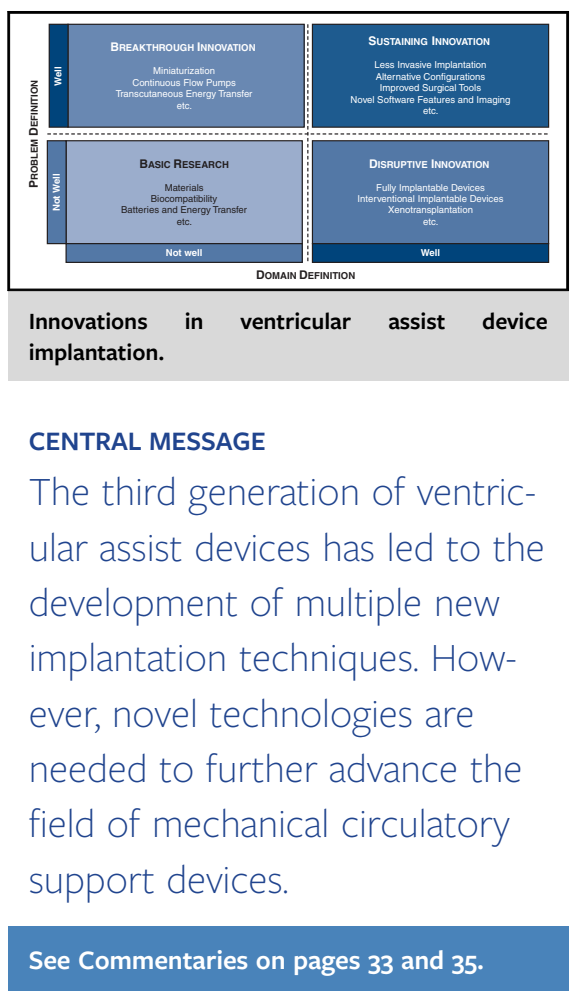

approach that provides good exposure for implanting the pump body compared with other techniques. To date, full sternotomy has been the most frequently used approach in all major scientific studies and thus has been researched the most. ${ }^{7}$ However, because the size of the pump body determines the size of the incision, the continuous miniaturization of third-generation VADs drove the development of less invasive surgery (LIS) procedures. ${ }^{3,6,8-10}$

For LIS left VAD (LVAD) implantation, most surgeons use a combination of a lateral thoracotomy and a hemisternotomy by tunneling the outflow graft through the left pleural space. ${ }^{3-6}$ Another viable alternative sternal-sparing strategy is the combination of an anterolateral thoracotomy and a right-sided upper thoracotomy. ${ }^{3,8,11}$ Benefits and weaknesses of LIS VAD implantation are summarized in Table 1.

A minor drawback of LIS VAD implantation is the withdrawal of the HeartWare HVAD (Medtronic Minneapolis, Minn) from the market in June 2021. ${ }^{12}$ When introduced in 2006, the HVAD was-especially compared with larger competitors, such as the Heart Mate II (Abbott, Abbott Park, Ill)—a highly innovative centrifugal pump that made 
TABLE 1. Benefits and drawbacks of less invasive ventricular assist device implantation

\begin{tabular}{ll}
\hline \multicolumn{1}{c}{ Benefits } & \multicolumn{1}{c}{ Drawbacks } \\
\hline Approved concept & No easy concomitant procedures \\
Easy to learn & Difficult in very obese patients \\
\hline Less sternal and wound infections & Limited space \\
$\begin{array}{l}\text { Fewer adhesions for future redo } \\
\text { procedures }\end{array}$ & Limited exposure \\
$\begin{array}{l}\text { Good cosmetic results and patient } \\
\text { preference }\end{array}$ \\
$\begin{array}{l}\text { Reduced right heart failure } \\
\text { Shorter intensive care unit stay }\end{array}$ \\
$\begin{array}{l}\text { Shorter stay on ward until } \\
\text { discharge }\end{array}$ \\
Economically beneficial
\end{tabular}

LIS implantation possible. ${ }^{3-5}$ Implantation via thoracotomy is also possible with Abbott's HeartMate 3, currently the most popular VAD; however, its slightly larger pump body and the unhandy click-in mechanism increase the difficulty of fitting the pump in smaller anatomic settings. ${ }^{5}$

Along with satisfying patients' desire for minimally invasive procedures, multiple studies suggest that LIS VAD implantation is associated with reduced right heart failure and use of blood products, decreased hospital length of stay, and overall improved survival, especially in INTERMACS 1 patients. It also is suggested to be a preferred method in reoperative procedures and seems to prevent large adhesions, an advantage in bridge-to-transplantation candidates. ${ }^{3,6,8,10,13,14}$ One criticism is that double-blinded large-scale trials are still missing for LIS implantation versus conventional sternotomy to scientifically prove these effects; however, the positive effects of conventional implantation have never been proven either.

\section{Alternative Sites and Techniques for Outflow Graft Anastomosis}

Conventionally, the outflow graft of the LVAD is anastomosed to the ascending aorta. ${ }^{3}$ This has several advantages, including providing the most physiologic blood flow and thus reduced flow turbulence, resulting in decreased adverse events. However, in certain anatomic settings (eg, porcelain aorta), alternative sites for outflow graft placement are required. Common alterative positions for outflow graft placement are the brachiocephalic trunk, subclavian artery, and descending aorta. Disadvantages of these techniques include the possible unbalanced hyperperfusion or hypoperfusion of the arm and head, flow disturbances, and surgical challenges. ${ }^{3,15-17}$ One alternative to conventional outflow graft placement involves tunneling the outflow graft through the transverse sinus with the aim of protecting the outflow graft in reoperative procedures, such as heart transplantation. ${ }^{18}$
A novel (albeit experimental) outflow graft anastomosis technique involves using the DaVinci robotic system to perform the anastomosis to the ascending aorta via a mini thoracotomy. ${ }^{19}$ Although this surgical approach is innovative, the necessary investments likely outweigh the advantages of this technique. Nevertheless, the feasibility of robotic-assisted VAD implantation has been demonstrated, and further advanced robotic-guided implantation might be a possible innovative approach in the future. ${ }^{19}$

\section{Exchange Procedure, Upgrade, and Explantation}

Owing to the increased implantation rates and the rising numbers of destination patients, VAD exchanges are progressively becoming a standard surgical procedure in all MCS programs. Serious device infection and thrombosis remain major complications of VAD therapy that need to be treated with a pump exchange if other treatments fail. ${ }^{3,7,20}$ It has been proven that upgrading the oldergeneration LVADs to a newer-generation device during LIS LVAD exchange is feasible and shows similar results as conventional exchange procedures. ${ }^{20}$ Given the advantages and technical improvements of the newer-generation pumps, the exchange procedure also provides an opportunity to upgrade patients to a superior generation of VADs with a preferable adverse event profile. ${ }^{20}$

Recovery of native cardiac function is a rarely achieved but highly aspired-to goal in VAD therapy. Surgical VAD explantation in cases of cardiac recovery can be a challenge given the high-risk patient cohort. In the past, sewing ring explantation and ventricular reconstruction was necessary for device explantation. Now several manufacturers have developed custom-made plugs for VAD explantation. Thus, VAD explantation solely through thoracotomy is recommended. An alternative approach to VAD explantation is decommissioning the device with operative or interventional outflow graft occlusion or banding via a mini thoracotomy. $^{21,22}$

\section{On- and Off-Pump Implantation Strategies}

To further decrease invasiveness, VAD implantations, exchanges, or upgrades potentially can be performed without use of the heart-lung machine. However, such procedures are widely considered unsafe because of the association with such risks as air embolism, stroke, blood loss, and right heart failure, which can be avoided by using on-pump techniques. Furthermore, on-pump techniques offer the opportunity to inspect the left ventricular cavity in detail and remove any endothelial, trabecular, or thromboembolic material. Thus, generally off-pump VAD implantation, exchange, or upgrade is recommended only in selected cases with contraindications for the use of a heart-lung machine, such as severe vascular calcifications or high risk of cerebral bleeding. ${ }^{3,23-25}$ 


\section{ALTERNATIVE CONFIGURATIONS: RVAD, BiVAD, VAD-TAH}

Implanting ventricular assist devices in alternative configurations is currently an off-label procedure. However, such configurations as single-ventricle right-sided VAD (RVAD) or the biventricular use of newer generation VADs have been described in the literature. Another offlabel therapy concept is the implantation of 2 VADs in the setting of a total artificial heart (TAH). ${ }^{26,27}$ As transplantation is quantitatively limited, cardiac replacement with a TAH offers an alternative surgical option as an ultima ratio concept in cases of severe biventricular failure. However, owing to the low number of TAH implantations performed worldwide, confidence in handling TAHs is rare in most cardiac centers. An alternative approach to TAH implantation is the use of 2 HeartMate 3 devices in a TAH configuration after cardiectomy (HM6). Although HM6 implantation has been proven feasible in several international centers, it remains an ultima ratio and off-label concept that needs further investigation. $^{26-28}$

\section{When to Use Novel Surgical Strategies and When Not to?}

Minimally invasive strategies are a trending development throughout all surgical and interventional specialties, and consequently and undoubtedly, LIS strategies are also the future of VAD implantation. In general, a conventional VAD implantation via a full sternotomy is a safe procedure in standard cases; however, especially in selected anatomic or physiological conditions (eg, right heart failure, reoperative procedures), LIS VAD implantation is a strong tool for improving outcomes.

With increasingly positive outcomes, physicians' confidence in VAD therapy is high, and thus the number of challenging cases will continue to increase. Surgeons are strongly recommended to invest time in learning LIS procedures and study alternative surgical approaches for the highly inhomogeneous group of patients with terminal heart failure.

Compared with the regulatory restrictions concerning new technologies, the regulatory oversight for the application of new surgical techniques is rather nonexistent. Owing to the possible complication of suboptimal device positioning, we recommend using an alternative technique only if optimal positioning of the inflow cannula and outflow graft can be reproducibly established. Yet it should be mentioned that not everything that surgically can be done needs to be done, and also that everything that is surgically feasible cannot be done by every surgeon. Thus, it is strongly recommended that challenging cases of VAD implantation be transferred to high-volume VAD centers for optimal treatment.

\section{OUTLOOK ON FUTURE DEVELOPMENTS}

As discussed earlier, there have been significant improvements in surgical technique with the introduction of a third generation of VADs. However, further surgical innovation cannot be expected with the currently available devices. Any new disruptive or breakthrough innovations will require new developments from the technological side. Over the last 5 years, the market for permanent VADs has been dominated by Abbott's HeartMate 3 and Medtronic's HVAD. Given that competition drives innovation, the now de facto monopoly status of Abbott's HeartMate 3 is potentially discouraging in the hope for novelty. Nevertheless, exciting technologies are currently in development or ready for market introduction, and discontinuation of the HVAD device might leave room for other innovative technologies to grow if funding can be secured.

Technological developments that are expected to bring breakthrough or disruptive innovation to surgical techniques include further miniaturization, novel technological features, fully implantable VADs, and improved tools for implantation. The various innovations in VAD implantation technique discussed in this report are listed in Figure 1.

\section{Miniaturization}

Further miniaturization of VADs will lead to even lesserinvasive implantation procedures. Small VADs, such as the MVAD (Medtronic) and Synergy (CircuLite, Saddle Brook, NJ) devices, offered smaller diameters and thus the possibility for smaller incisions; however, both devices were associated with increased thromboembolic adverse events and ultimately were taken off the market. ${ }^{29,30}$ Overcoming this challenge of increased thromboembolic risk for smaller devices would vastly increase the possibility of further reducing surgical trauma and also making long-term VAD therapy available for smaller children. Another option in the miniaturization of VADs is an interventionally implantable VAD. The implementation of these devices would be a disruptive innovation concerning implantation technique that could be similar to transcatheter aortic valve implantation techniques and decrease surgical trauma even further.

\section{Fully Implantable VADs}

The Achilles' heel of VADs remains the external driveline, which contributes to adverse events and unacceptance of the therapy form. Infections remain the major challenge in VAD therapy, which is associated with a relatively high rate of postoperative infections compared with cardiac transplantation. Transcutaneous energy transfer (TET) avoids this complication by eliminating the external driveline. Two patients have already been implanted with this innovative device, ${ }^{31}$ but large-scale trials and studies on 


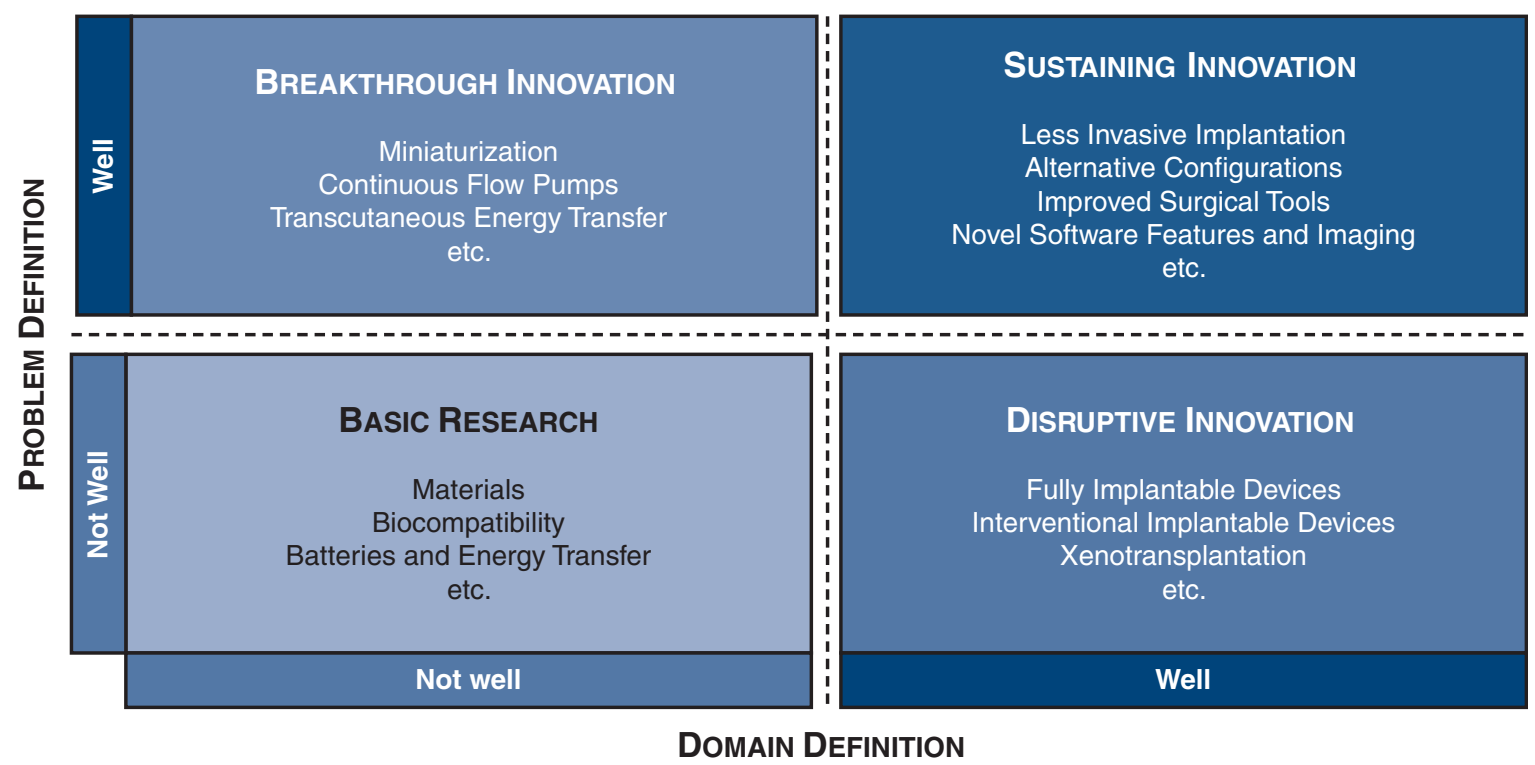

FIGURE 1. Four types of innovations in surgical implantation of ventricular assist devices. Original illustration, based on Henderson and Clark. ${ }^{2}$

the combination of this technology with established VADs are still needed to ensure the safety of this novel technology.

\section{Improved Surgical Tools}

Surgical demands change with new implantation techniques. Thus, with new techniques comes the need for new surgical tools to facilitate innovative approaches. One successful example is a new plug to ease HeartMate 3 explantation, which greatly simplifies the explantation procedure and avoids the need for excessive ventricular reconstruction after sewing ring removal. ${ }^{21,22}$ Other technological alterations in tools that facilitate implantation include an improved sewing ring with the ability to modify the insertion depth and angle of the inflow cannula, as well as an improved click-in mechanism for VAD insertion. A multitude of different coring tools have been developed and applied over the years; however, none of these devices fully eliminated the need for ventricular inspection and the further removal of trabeculae, which could possibly lead to suction events of pump thrombosis. Furthermore, improved intraoperative imaging, such as 3- and 4-dimensional echocardiography, has further optimized inflow cannula placement.

Other needed improvements to facilitate VAD implantation is the development of longer surgical tools which aim to ease less invasive implantation in obese patients as well as a technical option to improve deairing of the device. Other novel tools, such as automatic suturing devices, are currently in use in other surgical fields and might contribute to decreased implantation times for sewing ring and outflow graft anastomosis.

With the development of improved tools that facilitate implantation, such as better coring tools and deairing devices, further reduction in the use of surgical safety nets, such as the heart-lung machine, and safe off-pump implantation might be possible.

\section{Disruptive Innovation}

Despite technological advances, heart transplantation remains the gold standard for treating end-stage heart failure, and the risk of thrombosis and infection, along with dependence on technology, remain the major challenges with the use of current-generation VAD devices. ${ }^{8}$ However, transplantation is limited by a donor shortage, which is causing high mortality rates on the waiting lists if no alternative options are available. Thus, the permanent availability of VADs is a unique selling point and brands them as an attractive alternative to transplantation especially in emergency cases, as well as for patients who do not meet the criteria for heart transplantation.

A disruptive innovation in the field of permanent mechanical support would arise if VADs' unique selling point of permanent availability is met by a product with better biocompatibility that reduces the aforementioned VADassociated complications. Xenotransplantation might be such a disruptive innovative approach that may represent a giant leap forward in the treatment of terminal heart failure but would obliterate all the current innovations in VAD implantation. ${ }^{32}$

\section{CONCLUSIONS}

With beneficial adverse event profiles, LIS procedures are the future of heart failure surgery. The miniaturization of VADs has led to the development of several new surgical implantation strategies that are especially useful in challenging cases. However, surgical revolution is 
technology-driven, and given that the HeartMate 3 device was introduced in 2014, there is room for further breakthrough innovations. Significant innovations such as fully implantable devices, further miniaturization, novel technologies, and new/improved surgical tools, will lead to further advancements in implantation techniques.

\section{Conflict of Interest Statement}

Drs Hanke, Dogan, and Schmitto serve as consultants for Abbott, and Drs Dogan and Schmitto serve as consultants for Medtronic. All other authors reported no conflicts of interest.

The Journal policy requires editors and reviewers to disclose conflicts of interest and to decline handling or reviewing manuscripts for which they may have a conflict of interest. The editors and reviewers of this article have no conflicts of interest.

\section{References}

1. Schumpeter JA, Opie R, Elliott JE. The Theory of Economic Development: An Inquiry into Profits, Capital, Credit, Interest, and the Business Cycle. New Brunswick, NJ: Transaction Publishers; 1983.

2. Henderson RM, Clark KB. Architectural innovation: the reconfiguration of existing product technologies and the failure of established firms. Adm Sci Q. 1990; 35:9-30.

3. Hanke JS, Rojas SV, Avsar M, Haverich A, Schmitto JD. Minimally-invasive LVAD implantation: state of the art. Curr Cardiol Rev. 2015;11:246-51.

4. Schmitto JD, Molitoris U, Haverich A, Strueber M. Implantation of a centrifugal pump as a left ventricular assist device through a novel, minimized approach: upper hemisternotomy combined with anterolateral thoracotomy. J Thorac Cardiovasc Surg. 2012;143:511-3.

5. Schmitto JD, Krabatsch T, Damme L, Netuka I. Less invasive HeartMate 3 left ventricular assist device implantation. J Thorac Dis. 2018;10(Suppl 15): S1692-5.

6. McGee E Jr, Danter M, Strueber M, Mahr C, Mokadam NA, Wieselthaler G, et al. Evaluation of a lateral thoracotomy implant approach for a centrifugal-flow left ventricular assist device: the LATERAL clinical trial. J Heart Lung Transplant. 2019;38:344-51.

7. Mehra MR, Goldstein DJ, Uriel N, Cleveland JC Jr, Yuzefpolskaya M, Salerno C, et al. Two-year outcomes with a magnetically levitated cardiac pump in heart failure. $N$ Engl J Med. 2018;378:1386-95.

8. Carrozzini M, Bejko J, Gerosa G, Bottio T. Bilateral mini-thoracotomy approach for minimally invasive implantation of HeartMate 3. Artif Organs. 2019;43: 593-5.

9. Potapov E, Loforte A, Pappalardo F, Morshuis M, Schibilsky D, Zimpfer D, et al Impact of a surgical approach for implantation of durable left ventricular assist devices in patients on extracorporeal life support. J Card Surg. 2021;36: $1344-51$.

10. Riebandt J, Wiedemann D, Sandner S, Angleitner P, Zuckermann A, Schlöglhofer T, et al. Impact of less invasive left ventricular assist device implantation on heart transplant outcomes. Semin Thorac Cardiovasc Surg. February 17, 2021 [Epub ahead of print].

11. Popov AF, Hosseini MT, Zych B, Simon AR, Bahrami T. HeartWare left ventricular assist device implantation through bilateral anterior thoracotomy. Ann Thorac Surg. 2012;93:674-6.

12. Medtronic. Medtronic to stop distribution and sale of $\mathrm{HVAD}^{\mathrm{TM}}$ system. Available at: https://newsroom.medtronic.com/news-releases/news-release-details/ medtronic-stop-distribution-and-sale-hvadtm-system. Accessed June 22, 2021.
13. Mahr C, McGee E Jr, Cheung A, Mokadam NA, Strueber M, Slaughter MS, et al. Cost-effectiveness of thoracotomy approach for the implantation of a centrifugal left ventricular assist device. ASAIO J. 2020;66:855-61.

14. Pasrija C, Sawan MA, Sorensen E, Voorhees H, Shah A, Strauss E, et al. Less invasive left ventricular assist device implantation may reduce right ventricular failure. Interact Cardiovasc Thorac Surg. 2019;29:592-8.

15. Hanke JS, Rojas SV, Martens A, Schmitto JD. Minimally invasive left ventricular assist device implantation with outflow graft anastomosis to the innominate artery. J Thorac Cardiovasc Surg. 2015;149:e69-70.

16. Kawabori M, Critsinelis A, Kurihara C, Sugiura T, Morgan JA. Sternum-sparing HVAD implantation with attachment of the outflow graft to the descending aorta. ASAIO J. 2020;66:1006-13.

17. Maltais S, Danter MR, Haglund NA, Schmitto JD, Stulak JM. Nonsternotomy approaches for left ventricular assist device placement. Oper Tech Thorac Cardiovasc Surg. 2014;19:276-91.

18. Hanke JS, Rojas SV, Cvitkovic T, Wiegmann B, Horke A, Warnecke G, et al. First results of HeartWare left ventricular assist device implantation with tunnelling of the outflow graft through the transverse sinus. Interact Cardiovasc Thorac Surg. 2017; 25:503-8.

19. Khalpey Z, Sydow N, Paidy S, Slepian MJ, Friedman M, Cooper A, et al. Robotic-assisted implantation of ventricular assist device after sternectomy and pectoralis muscle flap. ASAIO J. 2014;60:742-3.

20. Hanke JS, Rojas SV, Dogan G, Feldmann C, Beckmann E, Deniz E, et al. First series of left ventricular assist device exchanges to HeartMate 3. Eur J Cardiothorac Surg. 2017;51:887-92.

21. Ricklefs M, Deodhar C, Chatterjee A, Feldmann C, Hanke JS, Heimeshoff J, et al. A new tool for an explantation strategy of HeartMate $3^{\mathrm{TM}}$ left ventricular assist device. J Thorac Dis. 2018;10(Suppl 15):S1825-8.

22. Hanke JS, Dogan G, Haverich A, Schmitto JD. First-in-man explantation of a HeartMate 3 left ventricular assist device via customized plug. Eur J Cardiothorac Surg. 2020;57:604-6.

23. Rojas SV, Avsar M, Khalpey Z, Hanke JS, Haverich A, Schmitto JD. Minimally invasive off-pump left ventricular assist device exchange: anterolateral thoracotomy. Artif Organs. 2014:38:539-42.

24. Iwanski J, Tran PL, Jerman C, Smith R, Kazui T, Khalpey Z. Off-pump left ventricular assist device exchange via re-do left mini-thoracotomy with original outflow graft preservation. Perfusion. 2017;32:179-82.

25. Saeed D, Sixt S, Albert A, Lichtenberg A. Minimally invasive off-pump implantation of HeartMate 3 left ventricular assist device. J Thorac Cardiovasc Surg. 2016;152:1446-7.

26. Daneshmand MA, Bishawi M, Milano CA, Schroder JN. The HeartMate 6. ASAIO J. 2020;66:e46-9.

27. Hanke JS, Dogan G, Haverich A, Schmitto JD. Implantation of two HeartMate 3s in the setting of a total artificial heart. Oper Tech Thorac Cardiovasc Surg. 2021; 26:67-80.

28. Strueber M, Schmitto JD, Kutschka I, Haverich A. Placement of 2 implantable centrifugal pumps to serve as a total artificial heart after cardiectomy. $J$ Thorac Cardiovasc Surg. 2012;143:507-9.

29. Hanke JS, Dogan G, Schmitto JD. Five years of therapy with the HeartWare MVAD. Artif Organs. 2021;45:637-8.

30. Klotz S, Meyns B, Simon A, Wittwer T, Rahmanian P, Schlensak C, et al. Partial mechanical long-term support with the CircuLite Synergy pump as bridge-totransplant in congestive heart failure. Thorac Cardiovasc Surg. 2010;58(Suppl 2):S173-8.

31. Pya Y, Maly J, Bekbossynova M, Salov R, Schueler S, Meyns B, et al. First human use of a wireless coplanar energy transfer coupled with a continuous-flow left ventricular assist device. J Heart Lung Transplant. 2019;38:339-43.

32. Längin M, Mayr T, Reichart B, Michel S, Buchholz S, Guethoff S, et al. Consistent success in life-supporting porcine cardiac xenotransplantation. Nature. 2018; 564:430-3.

Key Words: ventricular assist device, surgical implantation, innovation, less invasive, minimal invasive, upgrade, exchange 